\title{
Correction to: From indoor exposure to inhaled particle deposition: A multiphase journey of inhaled particles
}

\section{Kiao Inthavong (ه)}

School of Engineering, RMIT University, PO Box 71, Bundoora, 3083, Australia

(c) The Author(s) 2020, Corrected Publication November 2020

Correction to:

Kiao Inthavong

From indoor exposure to inhaled particle deposition: A multiphase journey of inhaled particles

Experimental and Computational Multiphase Flow 2020, 2(2): 59-78

https://doi.org/10.1007/s42757-019-0046-6

The article "From indoor exposure to inhaled particle deposition: A multiphase journey of inhaled particles" written by Kiao Inthavong, was originally published electronically on the publisher's internet portal (currently SpringerLink) on 22 October 2019 without open access. After publication in Volume 2, Issue 2, page 59-78, the author(s) decided to opt for Open Choice and to make the article an open access publication. Therefore, the copyright of the article has been changed to (C) The Author(s) 2020 and the article is forthwith distributed under the terms of the Creative Commons Attribution 4.0 International License (http://creativecommons.org/licenses/by/4.0/), which permits use, duplication, adaptation, distribution and reproduction in any medium or format, as long as you give appropriate credit to the original author(s) and the source, provide a link to the Creative Commons license, and indicate if changes were made.

The original article has been corrected and can be found at https://doi.org/10.1007/s42757-019-0046-6.

$\square$ kiao.inthavong@rmit.edu.au

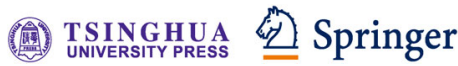

